\begin{tabular}{|l|l|}
\hline 2. To: (Receiving Organization) & 3. From: (Originating Organization) \\
Distribution & D. E. P] ace, SESC, H5-27 \\
\hline 5. Proj./Prog./Dept./Div.: & 6. Design Authority/ Design Agent/Cog \\
Tank 241-TX-111 & Engr.: J. Kupfer K. \\
\hline
\end{tabular}

8. Originator Remarks:

Approval and release.
4. Related EOT No.:

NA

7. Purchase Order No.:

NA

9. Equip./Component No.:

$\mathrm{NA}$

10. System/Bldg./Facility: NA

12. Major Assm. Dwg. No.: NA

13. Permit/Permit Application No.: NA

14. Required Response Date:

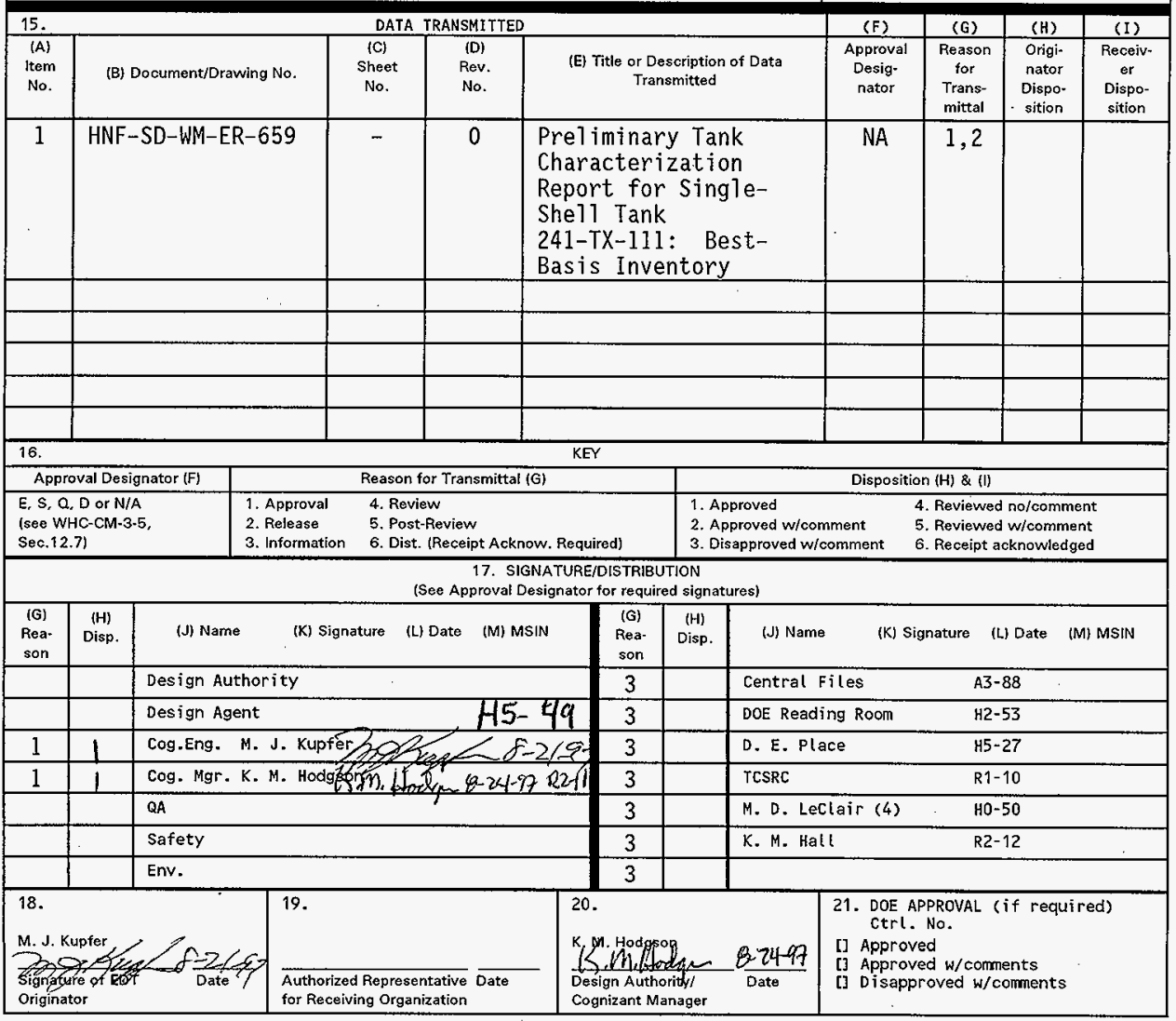




\title{
Preliminary Tank Characterization Report for Single-Shell Tank 241-TX-111: Best-Basis Inventory
}

\author{
D. E. Place
}

SGN Eurisys Services Corporation, Richland, WA 99352

U.S. Department of Energy Contract DE-AC06-96RL13200

$\begin{array}{lll}\text { EDT/ECN: } 617615 & \text { UC: } 712 \\ \text { Org Code: } 74610 \mathrm{kN} & \text { Charge Code: } & \text { N4G3A } \\ \text { B\&R Code: } & \text { EW3120074 } & \text { Total Pages: }\end{array}$

B\&R Code: EW3120074 Total Pages: $29 \mathrm{kN}$

Key Words: TCR, best-basis inventory

Abstract: An effort is underway to provide waste inventory estimates that will serve as standard characterization source terms for the various waste management activities. As part of this effort, an evaluation of available information for single-shell tank 241-TX-111 was performed, and a best-basis inventory was established. This work follows the methodology that was established by the standard inventory task.

TRADEMARK DISCLAIMER. Reference herein to any specific commercial product, process, or service by trade name, trademark, manufacturer, or otherwise, does not necessarily constitute or imply its endorsement, recommendation, or favoring by the United States Government or any agency thereof or its contractors or subcontractors.

Printed in the United States of America. To obtain copies of this document, contact: Document Control Services, P.0. Box 950, Mailstop H6-08, Richland WA 99352, Phone (509) 372-2420; Fax (509) 376-4989.

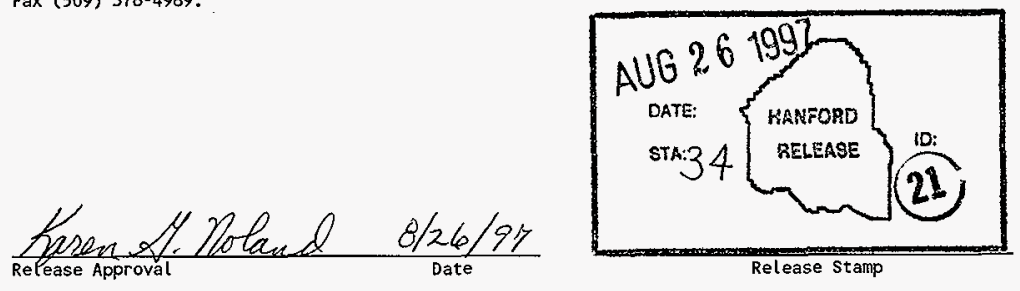

\section{Approved for Public Release}




\title{
PRELIMINARY TANK CHARACTERIZATION REPORT FOR SINGLE-SHELL TANK 241-TX-111: BEST-BASIS INVENTORY
}

July 1997

\author{
D. E. Place \\ SGN Eurisys Services Corporation \\ Richland, Washington
}

Prepared for

U.S. Department of Energy

Richland, Washington 
HNF-SD-WM-ER-659

Revision 0

This page intentionally left blank. 


\section{PRELIMINARY TANK CHARACTERIZATION REPORT FOR SINGLE-SHELL TANK 241-TX-111: BEST-BASIS INVENTORY}

This document is a preliminary Tank Characterization Report (TCR). It only contains the current best-basis inventory (Appendix D) for single-shell tank 241-TX-111. No TCRs have been previously issued for this tank, and current core sample analyses are not available. The best-basis inventory, therefore, is based on an engineering assessment of waste type, process flowsheet data, early sample data, and/or other available information.

The Standard Inventories of Chemicals and Radionuclides in Hanford Site Tank Wastes (Kupfer et al. 1997) describes standard methodology used to derive the tank-by-tank best-basis inventories. This preliminary TCR will be updated using this same methodology when additional data on tank contents become available.

\section{REFERENCE}

Kupfer, M. J., A. L. Boldt, B. A. Higley, K. M. Hodgson, L. W. Shelton, B. C. Simpson, and R. A. Watrous (LMHC), S. L. Lambert, and D. E. Place (SESC), R. M. Orme (NHC), G. L. Borsheim (Borsheim Associates), N. G. Colton (PNNL), M. D. LeClair (SAIC), R. T. Winward (Meier Associates), and W. W. Schulz (W'S Corporation), 1997, Standard Inventories of Chemicals and Radionuclides in Hanford Site Tank Wastes, HNF-SD-WM-TI-740, Rev. 0, Lockheed Martin Hanford Corporation, Richland, Washington. 
HNF-SD-WM-ER-659

Revision 0

This page intentionally left blank. 
HNF-SD-WM-ER-659

Revision 0

\section{APPENDIX D}

\section{EVALUATION TO ESTABLISH BEST-BASIS \\ INVENTORY FOR SINGLE-SHELL \\ TANK 241-TX-111}


HNF-SD-WM-ER-659

Revision 0

This page intentionally left blank.

D-2 
HNF-SD-WM-ER-659

Revision 0

APPENDIX D

\section{EVALUATION TO ESTABLISH BEST-BASIS INVENTORY FOR SINGLE-SHELL TANK 241-TX-111}

An effort is underway to provide waste inventory estimates that will serve as standard characterization source terms for the various waste management activities (Hodgson and LeClair 1996). As part of this effort, an evaluation of available information for single-shell tank 241-TX-111 was performed, and a best-basis inventory was established. This work, detailed in the following sections, follows the methodology that was established by the standard inventory task.

\section{D1.0 CHEMICAL INFORMATION SOURCES}

Available chemical and radiological inventory estimates for tank 241-TX-111 consist only of the inventory estimate generated by the Hanford Defined Waste (HDW) model (Agnew et al. 1996).

\section{D2.0 COMPARISON OF COMPONENT INVENTORY VALUES}

The tank 241-TX-111 chemical and radionuclide inventory predicted by the HDW model (Agnew et al. 1996) is provided in Table D2-1. The chemical species are reported without charge designation per the best-basis inventory convention.

Table D2-1. Hanford Defined Waste Model Prediction of Tank 241-TX-111 Inventory. (2 Sheets)

\begin{tabular}{|c|c|}
\hline Analyte & Hanford Defined Waste model ${ }^{a}(\mathrm{~kg})$ \\
\hline $\mathrm{Al}$ & 39,400 \\
\hline $\mathrm{Bi}$ & 1,960 \\
\hline $\mathrm{Ca}$ & 1,710 \\
\hline $\mathrm{Cl}$ & 7,430 \\
\hline $\mathrm{CO}_{3}$ & 28,200 \\
\hline $\mathrm{Cr}$ & 2,340 \\
\hline
\end{tabular}


HNF-SD-WM-ER-659

\section{Revision 0}

Table D2-1. Hanford Defined Waste Model Prediction of Tank 241-TX-111 Inventory. (2 Sheets)

\begin{tabular}{|c|c|}
\hline Analyte & Hanford Defined Waste model ${ }^{\mathrm{a}}(\mathrm{kg})$ \\
\hline $\mathrm{F}$ & 2,950 \\
\hline $\mathrm{Fe}$ & 2,060 \\
\hline $\mathrm{Hg}$ & 4.29 \\
\hline $\mathrm{K}$ & 2,150 \\
\hline $\mathrm{La}$ & $2.32 \mathrm{E}-04$ \\
\hline $\mathrm{Mn}$ & 154 \\
\hline $\mathrm{Na}$ & 268,000 \\
\hline $\mathrm{Ni}$ & 400 \\
\hline $\mathrm{NO}_{2}$ & 84,200 \\
\hline $\mathrm{NO}_{3}$ & 330,000 \\
\hline $\mathrm{OH}$ & 108,000 \\
\hline $\mathrm{Pb}$ & 223 \\
\hline $\mathrm{PO}_{4}$ & 24,800 \\
\hline $\mathrm{Si}$ & 2,240 \\
\hline $\mathrm{SO}_{4}$ & 25,200 \\
\hline $\mathrm{Sr}$ & $4.88 \mathrm{E}-05$ \\
\hline $\mathrm{U}$ & 2,820 \\
\hline $\mathrm{Zr}$ & 252 \\
\hline \multicolumn{2}{|l|}{ Radionuclide (Ci) } \\
\hline${ }^{137} \mathrm{Cs}$ & 236,000 \\
\hline${ }^{90} \mathrm{Sr}$ & 97,600 \\
\hline
\end{tabular}

${ }^{2}$ Agnew et al. (1996), radionuclides decayed to January 1, 1994. 
HNF-SD-WM-ER-659

Revision 0

\section{D3.0 COMPONENT INVENTORY EVALUATION}

\section{D3.1 CONTRIBUTING WASTE TYPES}

Information concerning the types of wastes contained in tank 241-TX-111 is not entirely consistent. The HDW model (Agnew et al. 1996) includes a sludge layer the is not reported by the Sort on Radioactive Waste Type (SORWT) model (Hill et al. 1995) or the waste tank summary report (Hanlon 1996).

The HDW model (Agnew et al. 1996) predicts that the tank contains first decontamination cycle (1C) waste from the bismuth phosphate process $(163 \mathrm{~kL}$ [43 kgal] of defined waste 1C2 sludge) and 1,238 kL (327 kgal) Supernatant Mixing Model 242-T Evaporator salt cake generated from 1965 until 1976 (SMMT2). Since the 1C wastes sent to this cascade were generated prior to 1955 , the coating wastes associated with the aluminumclad reactor fuel being processed were combined with the $1 \mathrm{C}$ waste in the underground storage tank (Anderson 1990).

The SORWT model (Hill et al. 1995) lists evaporator bottoms (EB), 1C (first cycle $\mathrm{BiPO}_{4}$ waste), and tri-butyl phosphate (TBP) (U Plant uranium recovery wastes) as the primary, secondary, and tertiary waste types respectively, but credits the entire tank $241-T X-111$ volume $(1,400 \mathrm{~kL}$ [370 kgal]) to salt cake with $34 \mathrm{~kL}(9 \mathrm{kgal})$ of interstitial liquid. Hanlon (1996) also indicates that the entire tank inventory is salt cake.

\section{D3.2 EVALUATION OF TECHNICAL FLOWSHEET INFORMATION}

Waste transaction records (Agnew et al. 1995) show that the cascade, consisting of tanks 241-TX-109 through 241-TX-112, received 1C wastes between the first quarter of 1949 and the fourth quarter of 1950 , between the second quarter of 1952 and the first quarter of 1954 , and during the third and fourth quarters of 1954. Waste transaction records indicate that a total of $19,455 \mathrm{~kL}(5,140 \mathrm{kgal})$ of combined $1 \mathrm{C} / \mathrm{cladding}$ waste $(1 \mathrm{C} / \mathrm{CW})$ was received into tank 241-TX-109 (Agnew et al. 1996). T Plant fuel processing during these periods consisted of approximately 1,473 MTU. The estimated 1C/CW waste volume based on the $\mathrm{BiPO}_{4}$ flowsheet (Schneider 1951) would be $21,726 \mathrm{~kL}(5,740 \mathrm{kgal})$, which is 12 percent higher than that indicated by the waste transaction records, but still in reasonably good agreement.

Most of the 1C wastes introduced into tank 241-TX-109 overflowed through the cascade piping to tanks 241-TX-110, 241-TX-111, and 241-TX-112. The waste transaction records show that $8,278 \mathrm{~kL}(2,187 \mathrm{kgal})$ of $1 \mathrm{C}$ waste was received in tank $241-\mathrm{TX}-111$. Most of the insoluble solids would have settled in the first two tanks of the cascade, but entrained solids would be expected in the overflow to tank 241-TX-111. The concentration of entrained solids in the 1C wastes received in tank $241-\mathrm{TX}-111$ would be expected to be 
low since the sludge level in tank $241-\mathrm{TX}-110$ was never large, and therefore, the residence time for settling would have been long.

Waste transaction records also indicate that $2,373 \mathrm{~kL}(627 \mathrm{kgal})$ of TBP waste was received from tank 241-TY-103 in the fourth quarter of 1954, of which $117 \mathrm{~kL}$ (31 kgal) was transferred to tank 241-TX-118. A small quantity of TBP waste (106 kL [28 kgall) was also received from tank 241-TX-110 via the cascade overflow in the first quarter of 1955 . Since the TBP wastes were stored in another tank for several months, no significant solids were expected in the transfer, and the contribution to the final composition of tank 241-TX-111 is expected to be small.

Beginning in the third quarter of 1970 and continuing until the second quarter of 1976 , tank 241-TX-111 received concentrated EB from the 242-T Evaporator and recycled supernates to the evaporator via the evaporator feed tank (241-TX-118). Salt cake formed as the concentrated salt solutions cooled and built up a large salt cake layer on top of previously existing sludge. Two airlift circulators were installed in the tank to facilitate evaporative cooling and prevent the formation of a crust which would have impeded evaporative heat removal. A small quantity (53 kL [14 kgal]) of evaporator feed was transferred from tank 241-S-102 in 1976 and early 1977. Supernate remaining in tank 241-TX-111 was removed from the tank in 1977. Salt well pumping of the interstitial liquid was accomplished in 1982 and 1983.

\section{D3.3 DETERMINATION OF WASTE TYPES}

The $1 \mathrm{C} / \mathrm{CW}$ volumes routed to tank $241-\mathrm{TX}-109$ through the tank $241-\mathrm{TX}-112$ cascade would result in $1,945 \mathrm{~kL}$ ( $514 \mathrm{kgal}$ ) of sludge (concentration factor [CF] of 10 based on tank 241-T-104). Most of this sludge volume would have been retained in the first two tanks of the cascade, but minor concentrations of entrained solids would still be expected in the overflow to tank 241-TX-111.

The HDW model predicts $163 \mathrm{~kL}$ (43 kgal) of 1C/CW sludge in tank 241-TX-111, based primarily on a sludge reading during the first quarter of 1970 , before the tank had been placed in EB service. As noted by Anderson (1990), there was a significant degree of uncertainty in these sludge level measurements. The HDW model also predicts $1,454 \mathrm{~kL}$ (384 kgal) of 1C sludge in tank 241-TX-109, $140 \mathrm{~kL}$ (37 kgal) in tank 241-TX-110, and none in tank 241-TX-112.

Assuming that $1,945 \mathrm{~kL}$ ( $514 \mathrm{kgal})$ of sludge resulted from the $1 \mathrm{C} / \mathrm{CW}$ waste routed to this four tank cascade, the inventory of tank $241-\mathrm{TX}-111 \mathrm{C}$ sludge could be as high as $352 \mathrm{~kL}$ ( $93 \mathrm{kgal}$ ) rather than the reported $163 \mathrm{~kL}$ ( $43 \mathrm{kgal}$ ). However, it is much more likely that any additional sludge inventory (if it actually exists) is contained in the first two tanks of the cascade. The $1 \mathrm{C}$ sludge inventory for tank $241-\mathrm{TX}-111$ is assumed to be $163 \mathrm{~kL}$ (43 kgal) to be consistent with the HDW model. 


\section{Revision 0}

Waste transaction records also show that tank $241-\mathrm{TX}-111$ received $4,300 \mathrm{~kL}$ (1,136 kgal) of concentrated evaporator waste between 1970 and 1976 . A substantial salt cake inventory would be expected to have been deposited on top of the sludge layer.

\section{D3.4 COMPOSITION OF TANK 241-TX-111 WASTE}

\section{D3.4.1 Composition of $1 \mathrm{C}$ Sludges}

Several tanks received $1 \mathrm{C} / \mathrm{CW}$ waste directly from $\mathrm{T}$ Plant including tanks 241-T-104, 241-T-107, 241-TX-109, 241-TX-113, 241-U-110, 241-TY-101, and 241-TY-103. Sample data are not available for solid layers in tanks $241-\mathrm{TX}-109$ or 241-TX-113. The $1 \mathrm{C}$ waste was mixed with substantial quantities of other wastes in tanks 241-TY-101, 241-TY-103, and 241-U-110, making it impossible to accurately determine the composition of the $1 \mathrm{C} / \mathrm{CW}$ waste sludge from these data. Two tanks (241-T-104 and 241-T-107) provide the best examples of $\mathrm{T}$ Plant $1 \mathrm{C} / \mathrm{CW}$ sludge composition. The composition of these two tanks, based on the corresponding TCRs (DiCenso et al. 1994 and Valenzuela and Jensen 1994), is provided in Table D3-1. The average of these two compositions will be used for estimating the sludge composition of tank 241-TX-111.

Table D3-1. Tank Characterization Report Concentrations for Tanks 241-T-104 and 241-T-107. (3 Sheets)

\begin{tabular}{|c|c|c|c|}
\hline Analyte & $\begin{array}{c}\text { Tank 241-T-104 } \\
(\mu \mathrm{g} / \mathrm{g})\end{array}$ & $\begin{array}{c}\text { Tank 241-T-107 } \\
(\mu \mathrm{g} / \mathrm{g})\end{array}$ & $\begin{array}{c}\text { Average } \\
\text { concentration }(\mu \mathrm{g} / \mathrm{g})\end{array}$ \\
\hline $\mathrm{Ag}$ & 6.4 & 7.37 & 6.9 \\
\hline $\mathrm{Al}$ & 16,200 & 16,300 & 16,200 \\
\hline $\mathrm{Bi}$ & 18,900 & 12,000 & 15,400 \\
\hline $\mathrm{Ca}$ & 1,450 & 760 & 1,100 \\
\hline $\mathrm{Cd}$ & 5.44 & 6.94 & 6.19 \\
\hline $\mathrm{Cl}$ & 670 & 540 & 605 \\
\hline $\mathrm{CO}$ & $<500$ & 14,800 & 7,680 \\
\hline $\mathrm{Cr}$ & 901 & 360 & 631 \\
\hline $\mathrm{F}$ & 8,570 & 11,400 & 9,980 \\
\hline $\mathrm{Fe}$ & 9,020 & 29,200 & 19,100 \\
\hline $\mathrm{Hg}$ & 0.127 & 0.14 & 0.13 \\
\hline $\mathrm{K}$ & 89.0 & 234 & 162 \\
\hline $\mathrm{La}$ & $<10.4$ & $<2$ & $<10$ \\
\hline
\end{tabular}


HNF-SD-WM-ER-659

Revision 0

Table D3-1. Tank Characterization Report Concentrations for Tanks 241-T-104 and 241-T-107. (3 Sheets)

\begin{tabular}{|c|c|c|c|}
\hline Analyte & $\begin{array}{c}\text { Tank 241-T-104 } \\
(\mu \mathrm{g} / \mathrm{g})\end{array}$ & $\begin{array}{c}\text { Tank } 241-\mathrm{T}-107 \\
(\mu \mathrm{g} / \mathrm{g})\end{array}$ & $\begin{array}{c}\text { Average } \\
\text { concentration }(\mu \mathrm{g} / \mathrm{g})\end{array}$ \\
\hline $\mathrm{Mn}$ & 61.8 & 213 & 137 \\
\hline $\mathrm{Na}$ & 64,500 & 130,200 & 97,400 \\
\hline $\mathrm{Ni}$ & 11.3 & 267 & 139 \\
\hline $\mathrm{NO}_{2}$ & 4,080 & 11,700 & 7,890 \\
\hline $\mathrm{NO}_{3}$ & 58,000 & 74,500 & 66,200 \\
\hline $\mathrm{OH}$ & NR & NR & NR \\
\hline $\mathrm{Pb}$ & NR & 649 & 649 \\
\hline $\mathrm{P}$ as $\mathrm{PO}_{4}$ & 75,700 & 98,400 & 87,100 \\
\hline Si & 6,520 & 6,050 & 6,280 \\
\hline $\mathrm{S}$ as $\mathrm{SO}_{4}$ & 3,830 & 9,810 & 6,820 \\
\hline $\mathrm{Sr}$ & 99.1 & 878 & 489 \\
\hline TOC & 706 & 963 & 835 \\
\hline $\mathrm{U}$ & 897 & 26,400 & 13,600 \\
\hline $\mathrm{Zr}$ & 67.5 & 93 & 80 \\
\hline Radionuclide & $\begin{array}{c}\text { Tank 241-T-104 } \\
(\mu \mathrm{Ci} / \mathrm{g})\end{array}$ & $\begin{array}{c}\text { Tank 241-T-107 } \\
(\mu \mathrm{Ci} / \mathrm{g})\end{array}$ & $\begin{array}{c}\text { Decayed average }{ }^{a} \\
(\mu \mathrm{Ci} / \mathrm{g})\end{array}$ \\
\hline${ }^{241} \mathrm{Am}$ & 0.0173 & 0.0141 & 0.0157 \\
\hline${ }^{14} \mathrm{C}$ & $<4.5 \mathrm{E}-05$ & $1.81 \mathrm{E}-04$ & $1.1 \mathrm{E}-04$ \\
\hline${ }^{60} \mathrm{Co}$ & $<3.0 \mathrm{E}-04$ & $<0.00199$ & $<9.85$ E-04 \\
\hline${ }^{134} \mathrm{Cs}$ & $\mathrm{NR}$ & $<0.00164$ & $<0.0012$ \\
\hline${ }^{137} \mathrm{Cs}$ & 0.199 & 12.0 & 5.96 \\
\hline${ }^{154} \mathrm{Eu}$ & 0.0041 & $<0.00463$ & 0.0038 \\
\hline${ }^{{ }^{155} \mathrm{Eu}}$ & 0.00342 & $<0.0149$ & 0.0030 \\
\hline${ }^{3} \mathrm{H}$ & $<3.38$ E-04 & 0.00124 & $5.9 \mathrm{E}-04$ \\
\hline${ }^{129} I$ & $<0.0464$ & NR & $<0.0464$ \\
\hline${ }^{237} \mathrm{~Np}$ & 0.137 & NR & 0.137 \\
\hline${ }^{238} \mathrm{Pu}$ & $<0.018$ & 0.144 & 0.072 \\
\hline${ }^{239 / 240} \mathrm{Pu}$ & 0.14 & 0.131 & 0.136 \\
\hline
\end{tabular}


Table D3-1. Tank Characterization Report Concentrations for Tanks 241-T-104 and 241-T-107. (3 Sheets)

\begin{tabular}{|c|c|c|c|}
\hline Radionuclide & $\begin{array}{c}\text { Tank 241-T-104 } \\
(\mu \mathrm{Ci} / \mathrm{g})\end{array}$ & $\begin{array}{c}\text { Tank 241-T-107 } \\
(\mu \mathrm{Ci} / \mathrm{g})\end{array}$ & $\begin{array}{c}\text { Decayed average } \\
(\mu \mathrm{Ci} / \mathrm{g})\end{array}$ \\
\hline${ }^{106} \mathrm{Ru}$ & $\mathrm{NR}$ & $<0.0757$ & $<0.038$ \\
\hline${ }^{79} \mathrm{Se}$ & $<1.75 \mathrm{E}-04$ & $\mathrm{NR}$ & $<1.75 \mathrm{E}-04$ \\
\hline${ }^{90} \mathrm{Sr}$ & 2.63 & 108 & 54.0 \\
\hline${ }^{99} \mathrm{Tc}$ & $5.79 \mathrm{E}-04$ & $\mathrm{NR}$ & $5.79 \mathrm{E}-04$ \\
\hline Density $(\mathrm{g} / \mathrm{mL})$ & 1.29 & 1.51 & 1.40 \\
\hline $\mathrm{Wt} \% \mathrm{H}_{2} \mathrm{O}$ & $70.5 \%$ & $56.0 \%$ & $63.2 \%$ \\
\hline
\end{tabular}

$\mathrm{NR}=$ Not reported

aDiCenso et al. (1994)

bValenzuela and Jensen (1994)

'Decayed to January 1, 1994, to match Hanford Defined Waste model.

\section{D3.4.2 Composition of 242-T Salt Cake}

Post-1965 operation of the 242-T Evaporator resulted in $22,672 \mathrm{~kL}(5,990 \mathrm{kgal})$ of salt cake which is contained in 26 underground storage tanks in the S, SX, U, T, TX, and TY Tank Farms. The HDW model refers to this salt cake as T2 Sltck on a global basis or as SMMT2 when calculated by the Supernatant Mixing Model (SMM) for an individual tank. The salt cake produced by the 242-T Evaporator from 1965 to 1976 will be referred to as T2 salt cake hereafter in this report. Ninety one percent of the T2 salt cake is contained in the TX Tank Farm. All tanks containing T2 salt cake also contain other waste types.

Only 8 tanks containing T2 salt cake have been core sampled (241-S-107, 241-U-102, 241-U-105, 241-U-107, 241-TX-107, 241-TX-116, 241-TY-102. and 241-TY-103). Only three of these tanks (241-U-102, 241-U-105, and 241-TX-116) have T2 salt cake layers large enough to be differentiated from the other tank wastes in core sample data.

T2 salt cake was formed in tanks 241-U-102 and 241-U-105 in 1975 through 1976. The core sampling of tanks $241-\mathrm{U}-102$ and $241-\mathrm{U}-105$ were performed in early 1996 . Based on the HDW model, segments 4,5 , and 6 for the two cores from tank 241-U-102 and segment 8 of two cores from tank 241-U-105 are expected to be representative of the T2 salt cake waste type. The recent analytical data should meet all Hanford Federal Facility Agreement and Consent Order (Tri-Party Agreement) Ecology (1994) requirements. Descriptions of the core sampling events and analytical data are available in the respective Tank Characterization Reports (Hu et al. 1997 and Brown and Franklin 1996). 
T2 salt cake was deposited in tank 241-TX-116 between 1966 and 1971 . The tank 241-TX-116 core sample was taken with the initial prototype of a rotary core sampler from 1976 to 1977 (Allen 1977). Sample recoveries were relatively poor. Additionally, analytical methods, data sets and quality assurance differed significantly from current practices.

However, this sample event provides the only available composition data for early production of the $\mathrm{T} 2$ salt cake waste type. Inclusion of an early $\mathrm{T} 2$ salt cake type is important since 242-T Evaporator feeds and operating practices changed over time. The analytical data are provided in a letter report (Horton 1977). Core segments 1 through 4 are expected to be representative $\mathrm{T} 2$ salt cake. It was necessary to correct the analytical results to a silica-free basis since approximately $95 \mathrm{MT}$ of diatomaceous earth $\left(92\right.$ percent $\mathrm{SiO}_{3}$ ) had been added to tank 241-TX-116. The silica from the diatomaceous earth had migrated into the top four core segments (approximately $203 \mathrm{~cm}$ [80 in.]) of the salt cake.

The composition data for tanks 241-U-102, 241-U-105, and 241-TX-116 are summarized in Table D3-2. The analytical results for tanks 241-U-102 and 241-U-105 are mass-weighted averages based on the mass of the partial core segment corresponding to each analytical result. Mass-weighted averages, rather than simple arithmetic averages, were calculated because the core segments were not of equal length and the mass of the partial core segments analyzed varied from approximately $30 \mathrm{~g}$ to $250 \mathrm{~g}$. Similarly, a massweighted average was created for the combination of the T2 salt cake in the two U Farm tanks (81.5\% tank 241-U-102 and 18.5\% tank 241-U-105). The analytical results for tank. 241-TX-116 core segments were simply averaged since the core segments were of equal length. The T2 salt cake prediction is the arithmetic average of the U Tank Farm and tank 241-TX-116 concentrations. The data for tank 241-TX-116 were intentionally given more emphasis (50 percent of the predicted concentration) in the generalized $\mathrm{T} 2$ salt cake prediction as it represents an operating period that is more applicable to the TX Tank Farm. The global HDW model composition for T2 salt cake (T2 SltCk) is included in the Table D3-2 for comparison.

Table D3-2. Composition of T2 Salt Cakes (3 Sheets).

\begin{tabular}{|c|c|c|c|c|c|c|}
\hline Analyte & $\begin{array}{c}\text { 241-U-102 } \\
\text { T2 salt cake } \\
\text { wt. avg., } \\
(\mu \mathrm{g} / \mathrm{g})\end{array}$ & $\begin{array}{c}\text { 241-U-105 } \\
\text { T2 salt cake } \\
\text { wt. avg., } \\
(\mu \mathrm{g} / \mathrm{g})\end{array}$ & $\begin{array}{c}\text { U Tank } \\
\text { Farm } \\
\text { T2 salt } \\
\text { cake } \\
\text { wt. avg. } \\
(\mu \mathrm{g} / \mathrm{g})\end{array}$ & $\begin{array}{c}\text { 241-TX-116 } \\
\text { T2 salt cake } \\
\text { mean }^{\mathrm{d}, \mathrm{e}} \\
(\mu \mathrm{g} / \mathrm{g})\end{array}$ & $\begin{array}{c}\text { T2 salt } \\
\text { cake } \\
\text { prediction }^{\mathrm{f}} \\
(\mu \mathrm{g} / \mathrm{g})\end{array}$ & $\begin{array}{c}\text { HDW } \\
\text { T2 SltCk } \\
(\mu \mathrm{g} / \mathrm{g})\end{array}$ \\
\hline $\mathrm{Ag}$ & 11.6 & 19.7 & 13.1 & $\mathrm{NR}$ & 13.1 & $\mathrm{NR}$ \\
\hline $\mathrm{Al}$ & 18,000 & 12,900 & 17,100 & 38,000 & 27,500 & 17,912 \\
\hline $\mathrm{Bi}$ & $<70.5$ & $<47.2$ & $<66.2$ & $\mathrm{NR}$ & $<66.2$ & 220.81 \\
\hline $\mathrm{Ca}$ & 308 & 253 & 298 & $\mathrm{NR}$ & 298 & 1,462 \\
\hline $\mathrm{Cd}$ & $<5.94$ & 12.8 & $<7.21$ & $\mathrm{NR}$ & $<7.21$ & $\mathrm{NR}$ \\
\hline $\mathrm{Cl}$ & 5,100 & 5,790 & 5,230 & $\mathrm{NR}$ & 5,230 & $3,327.8$ \\
\hline
\end{tabular}


Table D3-2. Composition of T2 Salt Cakes (3 Sheets).

\begin{tabular}{|c|c|c|c|c|c|c|}
\hline Analyte & $\begin{array}{c}241-\mathrm{U}-102 \\
\text { T2 salt cake } \\
\text { wt. avg., } \\
(\mu \mathrm{g} / \mathrm{g})\end{array}$ & 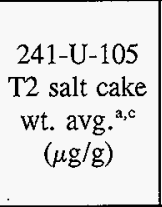 & $\begin{array}{c}\text { U Tank } \\
\text { Farm } \\
\text { T2 salt } \\
\text { cake } \\
\text { wt. avg.a } \\
(\mu \mathrm{g} / \mathrm{g})\end{array}$ & $\begin{array}{c}241-\mathrm{TX}-116 \\
\text { T2 salt cake } \\
\text { mean }^{\mathrm{d}, \mathrm{e}} \\
(\mu \mathrm{g} / \mathrm{g})\end{array}$ & $\begin{array}{c}\mathrm{T} 2 \text { salt } \\
\text { cake } \\
\text { prediction }^{\mathrm{f}} \\
(\mu \mathrm{g} / \mathrm{g})\end{array}$ & $\begin{array}{c}\text { HDW } \\
\text { T2 SitCk } \\
(\mu \mathrm{g} / \mathrm{g})\end{array}$ \\
\hline $\mathrm{CO}_{3}$ & 53,500 & 36,500 & 50,300 & 58,000 & 54,200 & 17,093 \\
\hline $\mathrm{Cr}$ & 2,310 & 2,100 & 2,270 & 353 & 1,310 & 4259.6 \\
\hline $\mathrm{F}$ & $<125$ & 1,110 & $<307$ & 3,540 & $<1,920$ & 930.79 \\
\hline $\mathrm{Fe}$ & 391 & 2,270 & 737 & 23,900 & 12,300 & 620.58 \\
\hline $\mathrm{Hg}$ & $\mathrm{NR}$ & NR & NA & NR & $\mathrm{NA}$ & 1.1338 \\
\hline $\mathrm{K}$ & 1750 & 1,470 & 1,700 & NR & 1,700 & 1060.7 \\
\hline $\mathrm{La}$ & $<35.2$ & 29.7 & $<34.2$ & NR & $<34.2$ & $1.0 \mathrm{E}-04$ \\
\hline $\mathrm{Mn}$ & 123 & 743 & 237 & NR & 237 & $160: 31$ \\
\hline $\mathrm{Na}$ & 262,600 & 220,500 & 254,800 & 166,700 & 210,800 & 192,764 \\
\hline $\mathrm{Ni}$ & 91.5 & 89.5 & 91.1 & NR & 91.1 & 405.82 \\
\hline $\mathrm{NO}_{2}$ & 56,700 & 40,100 & 53,600 & 7,840 & 30,700 & 46,096 \\
\hline $\mathrm{NO}_{3}$ & 284,700 & 395,700 & 305,200 & 308,700 & 306,946 & 268,197 \\
\hline $\mathrm{OH}$ & NR & NR & NA & NA & NA & 68,079 \\
\hline $\mathrm{Pb}$ & $<119$ & 214 & $<136$ & NR & $<136$ & 109.91 \\
\hline $\mathrm{P}$ as $\mathrm{PO}_{4}$ & 5,050 & 14,100 & 6,720 & 8,620 & 7,670 & $7,707.9$ \\
\hline $\mathrm{Si}$ & 152 & 232 & 167 & NR & 167 & $1,817.7$ \\
\hline $\mathrm{S}$ as $\mathrm{SO}_{4}$ & 17,900 & 8,350 & 16,200 & 16,400 & 16,300 & 13,823 \\
\hline $\mathrm{Sr}$ & $<7.04$ & $<4.72$ & $<6.61$ & $\mathrm{NR}$ & $<6.61$ & 0 \\
\hline TOC & 8,810 & 11,000 & 9,210 & NR & 9,210 & 5,191 \\
\hline $\mathrm{U}$ & $<353$ & 545 & $<388$ & NR & $<388$ & $2,174.3$ \\
\hline $\mathrm{Zr}$ & 10.8 & 45.4 & 17.2 & NR & 17.2 & 14.707 \\
\hline \multicolumn{7}{|c|}{ Radionuclide $^{\mathrm{h}}(\mu \mathrm{Ci} / \mathrm{g})$} \\
\hline${ }^{241} \mathrm{Am}$ & $<37.0$ & $<0.95$ & $<30.3$ & NR & $<30.3$ & 0.0285 \\
\hline${ }^{60} \mathrm{Co}$ & $<0.155$ & 0.086 & $<0.142$ & NR & $<0.142$ & 0.027 \\
\hline${ }^{134} \mathrm{Cs}$ & NR & $\mathrm{NR}$ & $\mathrm{NA}$ & $9.64 \mathrm{E}-04$ & $9.64 \mathrm{E}-04$ & 0.0016 \\
\hline${ }^{137} \mathrm{Cs}$ & 197 & 145 & 188 & 34.8 & 111 & 163.24 \\
\hline${ }^{154} \mathrm{Eu}$ & $<0.475$ & 0.61 & $<0.499$ & NR & $<0.499$ & 0.431 \\
\hline${ }^{155} \mathrm{Eu}$ & $<1.10$ & 0.82 & $<1.05$ & NR & $<1.05$ & 0.1849 \\
\hline
\end{tabular}




\section{Revision 0}

Table D3-2. Composition of T2 Salt Cakes (3 Sheets).

\begin{tabular}{|c|c|c|c|c|c|c|}
\hline Analyte & $\begin{array}{c}\text { 241-U-102 } \\
\text { T2 salt cake } \\
\text { wt. avg., } \\
(\mu \mathrm{g} / \mathrm{g})\end{array}$ & $\begin{array}{c}\text { 241-U-105 } \\
\text { T2 salt cake } \\
\text { wt. avg., } \\
(\mu \mathrm{g} / \mathrm{g})\end{array}$ & $\begin{array}{c}\text { U Tank } \\
\text { Farm } \\
\text { T2 salt } \\
\text { cake } \\
\text { wt. avg. } \\
(\mu \mathrm{g} / \mathrm{g})\end{array}$ & $\begin{array}{c}\text { 241-TX-116 } \\
\text { T2 salt cake } \\
\text { mean }^{\mathrm{d}, \mathrm{e}} \\
(\mu \mathrm{g} / \mathrm{g})\end{array}$ & $\begin{array}{c}\text { T2 salt } \\
\text { cake } \\
\text { prediction }^{\mathrm{f}} \\
(\mu \mathrm{g} / \mathrm{g})\end{array}$ & $\begin{array}{c}\text { HDW } \\
\text { T2 SltCk } \\
(\mu \mathrm{g} / \mathrm{g})\end{array}$ \\
\hline $\begin{array}{c}\text { Density } \\
(\mathrm{g} / \mathrm{mL})\end{array}$ & 1.66 & 1.73 & $1.70^{\mathrm{i}}$ & NR & 1.70 & 1.634 \\
\hline
\end{tabular}

HDW $=$ Hanford Defined Waste

NA $=$ Not applicable

$\mathrm{NR}=$ Not reported

${ }^{a}$ Weighted average based on the weight of each partial core segment analyzed

${ }^{b} \mathrm{Hu}$ et al. (1997)

${ }^{\circ}$ Brown and Franklin (1996)

${ }^{\mathrm{d}}$ Silica-free basis due to the addition of diatomaceous earth to this tank

${ }^{\mathrm{e}}$ Horton (1977)

${ }^{f}$ Average of U Tank Farm and tank 241-TX-116 data

${ }^{\mathrm{g}}$ Agnew et al. (1997)

${ }^{\text {h }}$ Decayed to January 1, 1994

${ }^{\mathrm{i}}$ A simple average is used for the density.

\section{D3.5 PREDICTED INVENTORY FOR TANK 241-TX-111}

The chemical and radionuclide inventory of tank 241-TX-111 can be estimated from the sludge and salt cake volumes ( $163 \mathrm{~kL}$ [43 kgal] and $1,238 \mathrm{~kL}$ [327 kgal], respectively), densities (1.4 and $1.7 \mathrm{~g} / \mathrm{mL}$ respectively), and the average of chemical/radionuclide concentrations calculated for $1 \mathrm{C} / \mathrm{CW}$ sludges and $\mathrm{T} 2$ salt cake wastes that have been analyzed. The resulting inventories are provided in Table D3-3. The inventories estimated by the HDW model (Agnew et al. 1996) are included in the table for comparison. 
HNF-SD-WM-ER-659

Revision 0

Table D3-3. Estimated Chemical and Radionuclide Inventory for Tank 241-TX-111. (2 Sheets)

\begin{tabular}{|c|c|c|c|c|}
\hline Analyte & $\begin{array}{c}1 \mathrm{C} / \mathrm{CW} \\
\text { sludge layer } \\
(\mathrm{kg})\end{array}$ & $\begin{array}{c}\text { T2 salt } \\
\text { cake layer }^{a} \\
(\mathrm{~kg})\end{array}$ & $\begin{array}{c}\text { TX-111 } \\
\text { inventory } \\
(\mathrm{kg})\end{array}$ & $\begin{array}{l}\text { HDW model } \\
\text { inventory } \\
(\mathrm{kg})\end{array}$ \\
\hline $\mathrm{Ag}$ & 1.57 & 27.6 & 29.2 & NR \\
\hline $\mathrm{A} 1$ & 3,700 & 58,000 & 61,700 & 39,400 \\
\hline $\mathrm{Bi}$ & 3,520 & $<139$ & $3,660^{\circ}$ & 1,960 \\
\hline $\mathrm{Ca}$ & 252 & 626 & 878 & 1,710 \\
\hline $\mathrm{Cd}$ & 1.41 & $<15.2$ & $<16.6$ & NR \\
\hline $\mathrm{Cl}$ & 138 & 11,000 & 11,100 & 7,430 \\
\hline $\mathrm{CO}_{3}$ & 1,750 & 114,000 & 115,700 & 28,200 \\
\hline $\mathrm{Cr}$ & 144 & 2,760 & 2,900 & 2,340 \\
\hline$F$ & 2,280 & $<4,040$ & $<6,320$ & 2,950 \\
\hline $\mathrm{Fe}$ & 4,350 & 25,900 & 30,300 & 2,060 \\
\hline $\mathrm{Hg}$ & 0.030 & NR & NA & 4.29 \\
\hline $\mathrm{K}$ & 36.8 & 3,570 & 3,610 & 2,150 \\
\hline $\mathrm{La}$ & $<2.3$ & $<72.0$ & $<74.3$ & $2.32 \mathrm{E}-04$ \\
\hline $\mathrm{Mn}$ & 31.3 & 499 & 530 & 154 \\
\hline $\mathrm{Na}$ & 22,200 & 443,500 & 465,700 & 268,000 \\
\hline $\mathrm{Ni}$ & 31.7 & 192 & 224 & 400 \\
\hline $\mathrm{NO}_{2}$ & 1,800 & 64,700 & 66,500 & 84,200 \\
\hline $\mathrm{NO}_{3}$ & 15,100 & 645,800 & 660,900 & 330,000 \\
\hline $\mathrm{OH}$ & NR & $\mathrm{NA}$ & NA & 108,000 \\
\hline $\mathrm{Pb}$ & 148 & $<287$ & $<435$ & 223 \\
\hline $\mathrm{P}$ as $\mathrm{PO}_{4}$ & 19,800 & 16,100 & 36,000 & 24,800 \\
\hline $\mathrm{Si}$ & 1,430 & 352 & 1,780 & 2,240 \\
\hline $\mathrm{S}$ as $\mathrm{SO}_{4}$ & 1,560 & 34,300 & 35,800 & 25,200 \\
\hline $\mathrm{Sr}$ & 111 & $<13.9$ & $<125$ & $4.88 \mathrm{E}-05$ \\
\hline TOC & 190 & 19,400 & 19,600 & 11,853 \\
\hline $\mathrm{U}$ & 3,110 & $<817$ & $<3,930$ & $2,820^{\circ}$ \\
\hline $\mathrm{Zr}$ & 18.3 & 36.2 & 54.5 & 252 \\
\hline
\end{tabular}




\section{Revision 0}

Table D3-3. Estimated Chemical and Radionuclide Inventory for Tank 241-TX-111. (2 Sheets)

\begin{tabular}{|c|c|c|c|c|}
\hline Radionuclide & $\begin{array}{c}\text { 1C/CW } \\
\text { sludge layer } \\
(\mathrm{Ci})\end{array}$ & $\begin{array}{c}\text { T2 salt } \\
\text { cake layer } \\
\text { (Ci) }\end{array}$ & $\begin{array}{c}\text { Total TX-111 } \\
\text { inventory } \\
\text { (Ci) }\end{array}$ & $\begin{array}{c}\text { HDW model } \\
\text { inventory } \\
(\mathrm{Ci})\end{array}$ \\
\hline${ }^{241} \mathrm{Am}$ & 3.58 & $<63,800$ & NA & NR \\
\hline${ }^{14} \mathrm{C}$ & 0.0257 & NR & NA & NR \\
\hline${ }^{60} \mathrm{Co}$ & $<0.225$ & $<299$ & NA & NR \\
\hline${ }^{134} \mathrm{Cs}$ & $<0.267$ & 2.03 & 2.03 & NR \\
\hline${ }^{137} \mathrm{Cs}$ & 1,360 & 234,000 & 235,400 & 236,000 \\
\hline${ }^{154} \mathrm{Eu}$ & 0.862 & $<1,050$ & NA & NR \\
\hline${ }^{155} \mathrm{Eu}$ & 0.678 & $<2,200$ & NA & NR \\
\hline${ }^{3} \mathrm{H}$ & 0.134 & NR & NA & NR \\
\hline${ }^{129} \mathrm{I}$ & $<10.6$ & NR & NA & NR \\
\hline${ }^{237} \mathrm{~Np}$ & 31.2 & NR & NA & NR \\
\hline${ }^{238} \mathrm{Pu}$ & 16.3 & NR & NA & NR \\
\hline${ }^{239 / 240} \mathrm{Pu}$ & 30.9 & NR & NA & NR \\
\hline${ }^{106} \mathrm{Ru}$ & $<8.68$ & NR & NA & NR \\
\hline${ }^{79} \mathrm{Se}$ & $<0.04$ & NR & NA & NR \\
\hline${ }^{90} \mathrm{Sr}$ & 12,300 & NR & NA & 97,600 \\
\hline${ }^{99} \mathrm{Tc}$ & 0.132 & NR & NA & NR \\
\hline${ }^{10} \mathrm{DW}$ & & & & \\
\hline
\end{tabular}

HDW = Hanford Defined Waste, Agnew et al. (1996)

NA $=$ Not applicable

$\mathrm{NR}=$ Not reported

${ }^{\mathrm{a}}$ Estimate calculated using the $\mathrm{T} 2$ salt cake prediction

'Radionuclides decayed to January 1, 1994

"The "less than" designator was not carried to the "TX-110 inventory" column because the salt cake contribution to the $\mathrm{Bi}$ inventory was insignificant compared to the sludge layer contribution. 
HNF-SD-WM-ER-659

Revision 0

\section{D3.6 COMPARISON OF TANK 241-TX-111 INVENTORY ESTIMATES}

The lack of sample-based inventory data adds considerable uncertainty to estimation of chemical and radionuclide inventories for tank 241-TX-111. The use of waste composition data from tanks 241-T-104, 241-T-107, 241-U-102, 241-U-105, and 241-TX-116 to represent the wastes in tank $241-T X-111$ is a reasonable approach. However, it should be noted that the operating history of tank $241-\mathrm{TX}-111$ is different from any other Hanford Site tank containing similar waste types.

The tank 241-TX-111 inventories predicted by the HDW model and the estimate based on waste analyses in other tanks are generally of the same order of magnitude, although the HDW is generally somewhat lower. Part of the explanation for this difference may be that the HDW model calculated density for the salt cake in tank $241-\mathrm{TX}-111$ is $1.41 \mathrm{~g} / \mathrm{cc}$ based on the sodium, aluminum, and hydroxide concentrations, which is a much lower density than is generally found when salt cakes are actually analyzed. The calculated density is used in determining the HDW model inventory for all analytes.

Aluminum. The estimated aluminum inventory is 57 percent higher than that predicted by the HDW model. Part of this difference is due to the low salt cake density calculated by the HDW model $(1.41 \mathrm{~g} / \mathrm{cc})$, as compared to the $1.7 \mathrm{~g} / \mathrm{mL}$ estimated based on the analytical results from tanks $241-\mathrm{U}-102$ and 241-U-105. Additionally, the tank 241-TX-116 analytical results show a much higher aluminum concentration. The estimated aluminum inventory will be used for the best-basis.

Bismuth. The HDW model seems to underestimate the bismuth inventory for $1 \mathrm{C} / \mathrm{CW}$ waste tanks. Part of this discrepancy results from the HDW model assumption that 27 percent of the bismuth is soluble. Another factor is that the total of the waste volume transactions for $1 \mathrm{C} / \mathrm{CW}$ wastes received in tank $241-\mathrm{TX}-109$ is about 12 percent lower than that predicted from the $\mathrm{BiPO}_{4}$ flowsheet.

Carbonate and Hydroxide. The estimated tank 241-TX-111 carbonate inventory is 4.1 times the HDW model inventory, whereas the estimated hydroxide inventory is only 64 percent of that predicted by the HDW model. The hydroxide ion in Hanford Site waste tanks is converted to carbonate by the absorption of carbon dioxide from the ambient air. The one mole of absorbed carbon dioxide will react with two moles of hydroxide ion to form one mole of carbonate ion. The rate is difficult to model at best, and is accelerated by use of airlift circulators such as those installed in tank 241 -TX-111. Conversion of $49,600 \mathrm{~kg}$ of hydroxide to carbonate would account for the difference in the carbonate inventories. The HDW model does not adequately account for the absorption of carbon dioxide from the atmosphere. 
Fluorides. The estimated fluoride inventory is about twice that predicted by the HDW model. This is likely the result of the HDW model assumptions that sodium fluoride is the only chemical compound containing fluoride and that it does not precipitate. The formation of insoluble fluoride compounds (such as sodium fluorophosphate) may be causing some fluoride to precipitate and remain in the tank.

Iron. The estimated iron inventory is skewed by the high iron concentration (2.4 wt\%) reported for tank 241-TX-116. A later analysis of the salt cake in tank 241-TX-116 (Schulz 1980) indicated very little insoluble material. The high iron concentration is not likely for a salt cake since iron is insoluble in alkaline solutions and would not have been present in the evaporator feeds in significant concentrations. Therefore, the HDW model provides a better best basis.

Nitrate. The estimated nitrate inventory is twice that predicted by the HDW model. Nearly all of the nitrate is associated with the salt cake. The HDW model salt cake inventory is predicted by the Supernatant Mixing Model (SMM), and it is, therefore, difficult to determine the cause of this discrepancy. The global HDW model T2 salt cake concentrations (see Table D3-2) are very reasonable, indicating that either the problem lies within the SMM model or that some feed inputs have been missed.

Sodium. The predicted HDW sodium inventory is about 58 percent that predicted from data for tanks 241-T-104 and 241-T-107. The HDW model density calculated by the $\mathrm{HDW}$ model is $1.41 \mathrm{~g} / \mathrm{cc}$, which is about 17 percent below that normally expected for a salt cake. The global HDW model T2 salt cake sodium concentration is very reasonable (see Table D3-2). Either there is an internal problem in the SMM model calculations or some feed inputs have been missed.

Total Hydroxide. Once the best-basis inventories were determined, the hydroxide inventory was calculated by performing a charge balance with the valences of other analytes. In some cases, this approach requires that other analyte (e.g., sodium or nitrate) inventories be adjusted to achieve the charge balance. During such adjustments, the number of significant figures is not increased. This charge balance approach is consistent with that used by Agnew et al. (1997).

Cesium-137 and Strontium-90. The heat load for tank 241-TX-111 has been estimated at $6,155 \mathrm{BTU} / \mathrm{h}$ (Kummerer 1995). This corresponds to a maximum of $270,000 \mathrm{Ci}$ ${ }^{90} \mathrm{Sr}\left(0.0228 \mathrm{BTU} / \mathrm{h} / \mathrm{Ci}{ }^{90} \mathrm{Sr}\right)$ or a maximum of $382,000 \mathrm{Ci}{ }^{137} \mathrm{Cs}\left(0.0161 \mathrm{BTU} / \mathrm{h} / \mathrm{Ci}{ }^{137} \mathrm{Cs}\right)$. About 62 percent of the heat load appears to be the result of ${ }^{137} \mathrm{Cs}$ based on the estimated ${ }^{137} \mathrm{Cs}$ inventory. There is consequently a strong possibility of higher ${ }^{137} \mathrm{Cs}$ and $/$ or ${ }^{90} \mathrm{Sr}$ inventories in tank $241-\mathrm{TX}-111$. The higher ${ }^{90} \mathrm{Sr}$ inventory estimated by the HDW model will be used as the best basis to better account for the tank heat load. 
HNF-SD-WM-ER-659

Revision 0

\section{D4.0 DEFINE THE BEST-BASIS AND ESTABLISH COMPONENT INVENTORIES}

Information about chemical, radiological, and/or physical properties is used to perform safety analyses, engineering evaluations, and risk assessment associated with waste management activities, as well as regulatory issues. These activities include overseeing tank farm operations and identifying, monitoring, and resolving safety issues associated with these operations and with the tank wastes. Disposal activities involve designing equipment, processes, and facilities for retrieving wastes and processing them into a form that is suitable for long-term storage. Chemical and radiological inventory information are generally derived using three approaches: (1) component inventories are estimated using the results of sample analyses, (2) component inventories are predicted using the HDW model based on process knowledge and historical information, or (3) a tank-specific process estimate is made based on process flowsheets, reactor fuel data, essential material usage, and other operating data. Not surprisingly, the information derived from these different approaches is often inconsistent.

An effort is underway to provide waste inventory estimates that will serve as the standard characterization for the various waste management activities (Hodgson and LeClair 1996). As part of this effort, an evaluation of available information for tank 241-TX-111 was performed including the following:

- T Plant $\mathrm{BiPO}_{4}$ reactor fuel processing to confirm $1 \mathrm{C} / \mathrm{CW}$ waste volumes transferred into the tanks $241-\mathrm{TX}-109$ through $241-\mathrm{TX}-112$ cascade and to predict the quantity of resulting sludge.

- Waste transactions and operating data to confirm that salt cake was retained in tank 241-TX-111.

- Composition data from two waste tanks (241-T-104 [DiCenso et al. 1994] and 241-T-107 [Valenzuela and Jensen 1994]) that are expected to have similar sludge compositions and three waste tanks (241-U-102 [Hu et al. 1997], 241-U-105 [Brown and Franklin 1996], and 241-TX-116 [Horton 1977]) that are expected to have similar salt cake compositions.

- An inventory estimate generated by the HDW model (Agnew et al. 1996)

Based on this evaluation, a best-basis inventory was developed. No analytical data are available for the sludge or salt cake remaining in tank 241-TX-111 because no samples have been taken. The estimated inventory was, therefore, based on the composition of the $1 \mathrm{C} / \mathrm{CW}$ wastes in tanks 241-T-104 and 241-T-107 and the T2 salt cakes in tanks 241-U-102, 241-U-105, and 241-TX-116 since the wastes in these tanks have actually been analyzed. The HDW model inventories were used when no other data were available or when analytical data were suspect. 
The waste in tank 241-TX-111 consists of combined $\mathrm{BiPO}_{4}$ first decontamination cycle (1C) and coating wastes generated by T Plant during processing of irradiated, aluminum-clad reactor fuel $(163 \mathrm{~kL}$ [43 kgal]) and salt cake produced by the $241-\mathrm{T}$ Evaporator $(1,238 \mathrm{~kL}$ [327 kgal]). The $1 \mathrm{C} / \mathrm{CW}$ sludge layer has been in contact with large volumes of supernates, including salt solutions with sodium hydroxide concentrations of up to 3 molar. Leaching of some sludge components may have occurred and remaining sludge may differ from that predicted from other tanks containing $1 \mathrm{C} / \mathrm{CW}$ wastes. The best-basis inventory for tank 241-TX-111 is presented in Tables D4-1 and D4-2. The inventory values reported in Tables D4-1 and D4-2 are subject to change. Refer to the Tank Characterization Database (TCD) for the most current inventory values.

Best-basis tank inventory values are derived for 46 key radionuclides (as defined in Section 3.1 of Kupfer et al. 1997), all decayed to a common report date of January 1, 1994. Often, waste sample analyses have only reported ${ }^{90} \mathrm{Sr},{ }^{137} \mathrm{Cs}$, ${ }^{239 / 240} \mathrm{Pu}$, and total uranium (or total beta and total alpha), while other key radionuclides such as ${ }^{60} \mathrm{Co},{ }^{99} \mathrm{Tc},{ }^{129} \mathrm{I},{ }^{154} \mathrm{Eu},{ }^{155} \mathrm{Eu}$, and ${ }^{241} \mathrm{Am}$, etc., have been infrequently reported. For this reason it has been necessary to derive most of the 46 key radionuclides by computer models. These models estimate radionuclide activity in batches of reactor fuel, account for the split of radionuclides to various separations plant waste streams, and track their movement with tank waste transactions. (These computer models are described in Kupfer et al. 1997, Section 6.1 and in Watrous and Wootan 1997.) Model generated values for radionuclides in any of 177 tanks are reported in the HDW Rev. 4 model results (Agnew et al. 1997). The best-basis value for any one analyte may be either a model result or a sample or engineering assessment-based result if available. (No attempt has been made to ratio or normalize model results for all 46 radionuclides when values for measured radionuclides disagree with the model.) For a discussion of typical error between model derived values and sample derived values, see Kupfer et al. 1997, Section 6.1.10.

Best-basis tables for chemicals and only four radionuclides $\left({ }^{90} \mathrm{Sr},{ }^{137} \mathrm{Cs}, \mathrm{Pu}\right.$ and $\left.\mathrm{U}\right)$ were being generated in 1996, using values derived from an earlier version (Rev. 3) of the HDW model. When values for all 46 radionuclides became available in Rev. 4 of the HDW model, they were merged with draft best-basis chemical inventory documents. Defined scope of work in fiscal year 1997 did not permit HDW Rev. 3 chemical values to be updated to HDW Rev. 4 chemical values. 
HNF-SD-WM-ER-659

Revision 0

Table D4-1. Best-Basis Inventory Estimates for Nonradioactive Components in Tank 241-TX-111 (Effective January 31, 1997). (2 Sheets)

\begin{tabular}{|c|c|c|c|}
\hline Analyte & $\begin{array}{l}\text { Total inventory } \\
(\mathrm{kg})\end{array}$ & $\begin{array}{c}\text { Basis } \\
(\mathrm{S}, \mathrm{M}, \mathrm{E} \text {, or } \mathrm{C})^{\mathrm{M}}\end{array}$ & Comment \\
\hline $\mathrm{Al}$ & 61,700 & $\mathrm{E}$ & \\
\hline $\mathrm{Bi}$ & 3,660 & $\mathrm{E}$ & $\begin{array}{l}\text { Concentration varies between } 1 \mathrm{C} \\
\text { wastes. }\end{array}$ \\
\hline $\mathrm{Ca}$ & 880 & E & \\
\hline $\mathrm{Cl}$ & 11,100 & E & \\
\hline TIC as $\mathrm{CO}_{3}$ & 116,000 & $\mathrm{E}$ & \\
\hline $\mathrm{Cr}$ & 2,900 & $E$ & \\
\hline F & $<6,320$ & $\mathrm{E}$ & \\
\hline $\mathrm{Fe}$ & 2,060 & $\mathrm{M}$ & \\
\hline $\mathrm{Hg}$ & 4.3 & $\mathrm{M}$ & \\
\hline $\mathrm{K}$ & 3,610 . & $\mathrm{E}$ & \\
\hline $\mathrm{La}$ & $<74.3$ & $\mathrm{E}$ & \\
\hline $\mathrm{Mn}$ & 530 & $\mathrm{E}$ & \\
\hline $\mathrm{Na}$ & 466,000 & $\mathrm{E}$ & \\
\hline $\mathrm{Ni}$ & 220 & $\mathrm{E}$ & $\begin{array}{l}\text { Concentration varies significantly } \\
\text { between } 1 \mathrm{C} \text { waste tanks. }\end{array}$ \\
\hline $\mathrm{NO}_{2}$ & 66,500 & $\mathrm{E}$ & \\
\hline $\mathrm{NO}_{3}$ & 660,900 & $\mathrm{E}$ & \\
\hline $\mathrm{OH}_{\text {TOTAL }}$ & 146,000 & $\mathrm{C}$ & \\
\hline $\mathrm{Pb}$ & 220 & $\mathrm{M} / \mathrm{E}$ & \\
\hline $\mathrm{P}$ as $\mathrm{PO}_{4}$ & 36,000 & $\mathrm{E}$ & $\begin{array}{l}\text { Concentration varies between } 1 \mathrm{C} \\
\text { waste tanks. }\end{array}$ \\
\hline $\mathrm{Si}$ & 1,800 & $\mathrm{E}$ & \\
\hline $\mathrm{S}$ as $\mathrm{SO}_{4}$ & 35,800 & $\mathrm{E}$ & \\
\hline $\mathrm{Sr}$ & $<125$ & $\mathrm{E}$ & $\begin{array}{l}\text { Inventory is likely to be }<2.5 \mathrm{~kg} \\
\text { based on maximum }{ }^{90} \mathrm{Sr} \text { inventory. }\end{array}$ \\
\hline TOC & 19,600 & E & \\
\hline $\mathrm{U}_{\text {TOTAL }}$ & 2,820 & M & $\begin{array}{l}\text { Concentration varies significantly } \\
\text { between } 1 \mathrm{C} \text { waste tanks. }\end{array}$ \\
\hline
\end{tabular}


HNF-SD-WM-ER-659

Revision 0

Table D4-1. Best-Basis Inventory Estimates for Nonradioactive Components in Tank 241-TX-111 (Effective January 31, 1997). (2 Sheets)

\begin{tabular}{|c|c|c|c|}
\hline Analyte & $\begin{array}{c}\text { Total inventory } \\
(\mathrm{kg})\end{array}$ & $\begin{array}{c}\text { Basis } \\
(\mathrm{S}, \mathrm{M}, \mathrm{E}, \text { or } \mathrm{C})^{1}\end{array}$ & Comment \\
\hline $\mathrm{Zr}$ & 54 & $\mathrm{E}$ & \\
\hline
\end{tabular}

${ }^{1} \mathrm{~S}=$ Sample-based

$\mathrm{M}=$ Hanford Defined Waste model-based, Agnew et al. (1996)

$\mathrm{E}=$ Engineering assessment-based

$\mathrm{C}=$ Calculated by charge balance; includes oxides as hydroxides, not including $\mathrm{CO}_{3}, \mathrm{NO}_{2}, \mathrm{NO}_{3}, \mathrm{PO}_{4}, \mathrm{SO}_{4}$, and $\mathrm{SiO}_{3}$. 
Revision 0

Table D4-2. Best-Basis Inventory Estimate $\mathrm{f} r$ Radioactive Components in Tank 241-TX-111 Decayed to January 1, 1994 (Effective January 31, 1997). (2 Sheets)

\begin{tabular}{|c|c|c|c|}
\hline Analyte & $\begin{array}{l}\text { Total Inventory } \\
\text { (Ci) }\end{array}$ & $\begin{array}{c}\text { Basis } \\
(\mathrm{S}, \mathrm{M} \text {, or } \mathrm{E})^{1}\end{array}$ & Comment \\
\hline${ }^{3} \mathrm{H}$ & 186 & M & \\
\hline${ }^{14} \mathrm{C}$ & 25.8 & M & \\
\hline${ }^{59} \mathrm{Ni}$ & 1.93 & M & \\
\hline${ }^{60} \mathrm{Co}$ & 29.3 & M & \\
\hline${ }^{63} \mathrm{Ni}$ & 189 & M & \\
\hline${ }^{79} \mathrm{Se}$ & 2.89 & $M$ & \\
\hline${ }^{90} \mathrm{Sr}$ & $1.03 \mathrm{E}+05$ & $M$ & \\
\hline${ }^{90} \mathrm{Y}$ & $1.03 \mathrm{E}+05$ & $\mathrm{E} / \mathrm{M}$ & \\
\hline${ }^{93 \mathrm{~m}} \mathrm{Nb}$ & 10.3 & $\mathrm{M}$ & \\
\hline${ }^{93} \mathrm{Zr}$ & 14.2 & $\mathrm{M}$ & \\
\hline${ }^{99} \mathrm{Tc}$ & 184 & $M$ & \\
\hline${ }^{106} \mathrm{Ru}$ & 0.00582 & $M$ & \\
\hline${ }^{113 \mathrm{~m}} \mathrm{Cd}$ & 73.6 & $M$ & \\
\hline${ }^{125} \mathrm{Sb}$ & 128 & $M$ & \\
\hline${ }^{126} \mathrm{Sn}$ & 4.36 & M & \\
\hline${ }^{129} \mathrm{I}$ & 0.354 & $M$ & \\
\hline${ }^{134} \mathrm{Cs}$ & 2.0 & E & \\
\hline${ }^{137} \mathrm{~m} \mathrm{Ba}$ & 223,000 & $E$ & Based on $137 \mathrm{Cs}$ \\
\hline${ }^{137} \mathrm{Cs}$ & 235,400 & $\mathrm{E}$ & \\
\hline${ }^{151} \mathrm{Sm}$ & 10,200 & $\mathrm{M}$ & \\
\hline${ }^{152} \mathrm{Eu}$ & 3.89 & $M$ & \\
\hline${ }^{154} \mathrm{Eu}$ & 507 & M & \\
\hline${ }^{155} \mathrm{Eu}$ & 233 & $M$ & \\
\hline${ }^{226} \mathrm{Ra}$ & $1.34 \mathrm{E}-04$ & $M$ & \\
\hline${ }^{227} \mathrm{Ac}$ & 8.66 E-04 & $M$ & \\
\hline${ }^{228} \mathrm{Ra}$ & 0.197 & $M$ & \\
\hline${ }^{229} \mathrm{Th}$ & 0.00456 & $\mathrm{M}$ & \\
\hline${ }^{231} \mathrm{~Pa}$ & 0.00384 & M & \\
\hline
\end{tabular}


HNF-SD-WM-ER-659

Revision 0

Table D4-2. Best-Basis Inventory Estimate $\mathrm{f} r$ Radioactive Components in Tank 241-TX-111 Decayed to January 1, 1994 (Effective January 31, 1997). (2 Sheets)

\begin{tabular}{|c|c|c|c|}
\hline Analyte & $\begin{array}{c}\text { Total Inventory } \\
(\mathrm{Ci})\end{array}$ & $\begin{array}{c}\text { Basis } \\
(\mathrm{S}, \mathrm{M}, \text { or E) }\end{array}$ & Comment \\
\hline${ }^{232} \mathrm{Th}$ & 0.0121 & $\mathrm{M}$ & \\
\hline${ }^{232} \mathrm{U}$ & 0.985 & $\mathrm{M}$ & \\
\hline${ }^{233} \mathrm{U}$ & 3.78 & $\mathrm{M}$ & \\
\hline${ }^{234} \mathrm{U}$ & 3.19 & $\mathrm{M}$ & \\
\hline${ }^{235} \mathrm{U}$ & 0.138 & $\mathrm{M}$ & \\
\hline${ }^{236} \mathrm{U}$ & 0.0509 & $\mathrm{M}$ & \\
\hline${ }^{237} \mathrm{~Np}$ & 0.661 & $\mathrm{M}$ & \\
\hline${ }^{238} \mathrm{Pu}$ & 1.31 & $\mathrm{M}$ & \\
\hline${ }^{238} \mathrm{U}$ & 3.41 & $\mathrm{M}$ & \\
\hline${ }^{239} \mathrm{Pu}$ & 52.1 & $\mathrm{M}$ & \\
\hline${ }^{240} \mathrm{Pu}$ & 8.15 & $\mathrm{M}$ & \\
\hline${ }^{241} \mathrm{Am}$ & 53.1 & $\mathrm{M}$ & \\
\hline${ }^{241} \mathrm{Pu}$ & 85.9 & $\mathrm{M}$ & \\
\hline${ }^{242} \mathrm{Cm}$ & 0.15 & $\mathrm{M}$ & \\
\hline${ }^{242} \mathrm{Pu}$ & $4.71 \mathrm{E}-04$ & $\mathrm{M}$ & \\
\hline${ }^{243} \mathrm{Am}$ & 0.0019 & $\mathrm{M}$ & \\
\hline${ }^{243} \mathrm{Cm}$ & 0.0138 & $\mathrm{M}$ & \\
\hline${ }^{244} \mathrm{Cm}$ & 0.123 & $\mathrm{M}$ & \\
\hline${ }^{15} \mathrm{~S}$ & & & \\
\hline
\end{tabular}

${ }^{1} \mathrm{~S}=$ Sample-based

$\mathrm{M}=$ Hanford Defined Waste model-based, Agnew et al. (1997)

$\mathrm{E}=$ Engineering assessment-based

NR $=$ Not Reported. 
HNF-SD-WM-ER-659

Revision 0

\section{D5.0 APPENDIX D REFERENCES}

Agnew, S. F., R. A. Corbin, T. B. Duran, K. A. Jurgensen, T. P. Ortiz, and B. L. Young, 1995, Waste Status and Transaction Record Summary (WSTRS Rev. 2), WHC-SD-WM-TI-615, -614, -669, -689, Rev. 2, Los Alamos National Laboratory, Los Alamos, New Mexico.

Agnew, S. F., J. Boyer, R. A. Corbin, T. B. Duran, J. R. FitzPatrick, K. A. Jurgensen, T. P. Ortiz, and B. L. Young, 1996, Hanford Tank Chemical and Radionuclide Inventories: HDW Model Rev. 3, LA-UR-96-858, Los Alamos National Laboratory, Los Alamos, New Mexico.

Agnew, S. F., J. Boyer, R. A. Corbin, T. B. Duran, J. R. FitzPatrick, K. A. Jurgensen, T. P. Ortiz and B. L. Young, 1997, Hanford Tank Chemical and Radionuclide Inventories: HDW Model Rev. 4, LA-UR-96-3860, Los Alamos National Laboratory, Los Alamos, New Mexico.

Allen, G. K., 1977, Salt Cake and Sludge Characterization Program 241-TX-116 Hot Test and Subsequent Cold Test Results, RHO-CD-3, Rockwell Hanford Operations, Richland, Washington.

Anderson, J. D., 1990, A History of the 200 Area Tank Farms, WHC-MR-0132, Westinghouse Hanford Company, Richland, Washington.

Brown, T. M., and J. D. Franklin, 1996, Tank Characterization Report for Single-Shell Tank 241-U-105, WHC-SD-WM-ER-617, Rev. 0, Westinghouse Hanford Company, Richland, Washington.

DiCenso, A. T., L. C. Amato, J. D. Franklin, G. L. Nuttall, K. W. Johnson, and B. C. Simpson, 1994, Tank Characterization Report for Single-Shell Tank 241-T-104, HNF-WESD-WM-ER-372, Rev. 0, Westinghouse Hanford Company, Richland, kN Washington.

Ecology, EPA, and DOE, 1994, Hanford Federal Facility Agreement and Consent Order, as amended, Washington State Department of Ecology, U.S. Environmental Protection Agency, and U.S. Department of Energy, Olympia, Washington.

Hanlon, B. M., 1996, Waste Tank Summary Report for Month Ending August 31, 1996, WHC-EP-0182-101, Westinghouse Hanford Company, Richland, Washington.

Hill, J. G., G. S. Anderson, and B. C. Simpson, 1995, The Sort on Radioactive Waste Type Model: A Method to Sort Single-Shell Tanks into Characteristic Groups, PNL-9814, Rev. 2, Pacific Northwest Laboratory, Richland, Washington. 
Hodgson, K. M. and M. D. LeClair, 1996, Work Plan for Defining A Standard Inventory Estimate for Wastes Stored in Hanford Site Underground Tanks, WHC-SD-WM-WP-311, Rev. 1, Lockheed Martin Hanford Corporation, Richland, Washington.

Horton, J. E., 1977, Physical and Chemical Characterization of Tank 116-TX, Letter to G. K. Allen, Atlantic Richfield Hanford Company, Richland, Washington.

Hu, T. A., L. C. Amato, R. T. Winward, and R. D. Cromar, 1997, Tank Characterization Report for Single-Shell Tank 241-U-102, HNF-SD-WM-ER-618, Rev. 0, Lockheed Martin Hanford Corporation, Richland, Washington.

Kummerer, M., 1995, Heat Removal Characteristics of Waste Storage Tanks, WHC-SD-WM-SARR-010, Rev. 1, Westinghouse Hanford Company, Richland, Washington.

Kupfer, M. J., A. L. Boldt, B. A. Higley, K. M. Hodgson, L. W. Shelton, B. C. Simpson, and R. A. Watrous (LMHC), S. L. Lambert, and D. E. Place (SESC), R. M. Orme (NHC), G. L. Borsheim (Borsheim Associates), N. G. Colton (PNNL), M. D. LeClair (SAIC), R. T. Winward (Meier Associates), and W. W. Schulz (W'2 S Corporation), 1997, Standard Inventories of Chemicals and Radionuclides in Hanford Site Tank Wastes, HNF-SD-WM-TI-740, Rev. 0, Lockheed Martin Hanford Corporation, Richland, Washington.

Schneider, K. L., 1951, Flow Sheets and Flow Diagrams of Precipitation Separations Process, HW-23043, Hanford Atomic Products Operation, Richland, Washington.

Schulz, W. W., 1980, Removal of Radionuclides from Hanford Defense Waste Solutions, RHO-SA-51, Rockwell Hanford Operations, Richland, Washington.

Valenzuela, B. D., and L. Jensen, 1994, Tank Characterization Report for Single-Shell Tank 241-T-107, WHC-SD-WM-ER-382, Rev. 0, Westinghouse Hanford Company, Richland, Washington.

Watrous, R. A., and D. W. Wootan, 1997, Activity of Fuel Batches Processed Through Hanford Separations Plants, 1944 Through 1989, HNF-SD-WM-TI-794, Rev. 0, Lockheed Martin Hanford Corporation, Richland, Washington. 